\title{
AN UPDATE ON THE PROBLEM OF SMALL COMETS
}

\author{
J.C. BRANDT \\ Laboratory for Atmospheric and Space Physics, University of Colorado, Boulder CO, \\ $U S A$ \\ AND \\ M.F. A'HEARN \\ Department of Astronomy, University of Maryland, College Park MD, USA
}

The evidence for a major population of small comets (SCs) is summarized in two steps. First, we briefly summarize our previous work. Second, we describe recent work that continues to find SCs. When new capabilities become available that can detect SCs, we expect to find them and this is occurring. At present, their detection is a haphazard process and we advocate a dedicated, optimized search program.

We define SCs as those icy bodies (i.e., sublimating bodies) with radius less than 1000 meters. Often the existence of SCs is inferred from effects ascribed to them. These "effects" include: lunar cratering; cratering on Ganymede; excess interplanetary hydrogen; delivery of volatile materials to the terrestrial planets; and a source of fragile bodies entering the Earth's atmosphere. While some of these indirect methods support the existence of SCs, direct observations are clearly preferred.

For our previous work on this subject we refer to (Brandt et al., 1996; Brandt et al., 1997). We found that SCs are often detected when special circumstances produce intrinsic or apparent brightenings. An example of the former is the detection of 15 comets very near the Sun by coronagraphs on the SOLWIND and SMM (Solar Maximum Mission) spacecraft. These comets are too faint to be detected when passing the Earth's orbit, but brighten considerably at very small heliocentric distances (see Marsden, 1989). These comets are probably part of the Kreuz group. Also note that sungrazing is a normal end state for many comet orbits (Asher et al., 1994).

Examples of the latter situation occur when comets come very close to Earth. Comet $7 \mathrm{P} /$ PonsWinnecke in 1927 has radii of 310 or 210 meters for assumed albedos of 0.05 or 0.10 , respectively (see Yeomans, 1991). Comet C/1983 J1 (Sugano-Saigusa-Fujikawa) has a radius of 370 meters as determined by infrared, visual, and radar studies (Hanner et al., 1987). Also, Comet 41P/TuttleGiacobini-Kresák has a radius of 800 meters for an albedo of 0.02 as reported by Roemer (1966). Indeed, Roemer noted: "The nuclei of most periodic comets appear to be less than $10 \mathrm{~km}$ in radius, and many may have radii of less than $1 \mathrm{~km}$ " (emphasis added).

We have also noted that an optimum search strategy should be based on the unique properties of comets: an extended source, with most of the light coming from the coma rather than the nucleus; motion with respect to the background stars; and characteristic spectral signature. Most existing search programs are designed to detect moving point sources and hence, are not detecting SCs efficiently.

The recent work consists of two parts. First, another spacecraft, SOHO, with coronagraphs is in operation. The SOHO/LASCO observers have found more than thirty comets very near the Sun and reported them in IAU Circulars. Second, research using the Planetary Camera (PC) of the Hubble Space Telescope's WF/PC 2 has yielded two more SCs. For an assumed albedo of 0.04 and assumptions about the phase law Lamy et al. (1997a,b) find radii of 340 meters for comet 45P/Honda-Mrkos-Pajdušáková and 600 meters for comet 46P/Wirtanen. They conclude that comet $46 \mathrm{P} /$ Wirtanen "...belongs to a class of small, highly active nuclei...."

We conclude that the existence of SCs is not in doubt, but their number and properties are very uncertain. Reasonable estimates place the number of 250-meter radius SCs in a shell between 1 and $2 \mathrm{AU}$ heliocentric distance in the range 16 to 800 . The distribution function $f(r) \propto r^{-3}$ (differential) probably holds well below 1000 meters, perhaps down to $\sim 100$ meters before turning 
over. The turnover almost certainly does not occur at 1000 meters, the size at which our traditional methods of detection begin to fail. What is the origin of the SCs? Were they formed at the same time as the rest of the comets and simply represent the small radius/low mass part of the original distribution function? Are they largely the result of fragmentation processes that seem to account for the SCs seen very near the Sun? Or, do they originate in a variety of processes?

Because SCs are intrinsically faint, the search program must be optimized for efficiency. If the goal is to characterize SCs down to (say) 250 meters radius, we may need to search down to 100 meters radius because generally the radius is not observed directly.

When a suitable search program for SCs is completed, we can assess their utility as indicators of processes in the formation of the solar system, use them as clues to comet splitting events, and evaluate their potential importance in the interaction between planets and small bodies.

Finally, we note that our SCs are not related to the house-sized masses of water proposed by Frank and Sigwarth (1997a,b,c,d) to be impacting the Earth's upper atmosphere in large numbers. Sometimes these objects are called comets, but they are not. They don't sublimate to form comas or tails. Inappropriate use of a well-known word can only further confuse a contentious debate.

\section{References}

Asher, D.J., Bailey, M.E., Hahn, G., and Steel, D.I., (1994) Asteroid 5335 Damocles and its Implications for Cometary Dynamics, MNRAS, 267, 26-42

Brandt, J.C., A'Hearn, M.F.,Randall, C.E., Schleicher, D.G., Shoemaker, E.M., and Stewart, A.I.F. (1996) On the Existence of Small Comets and their Interactions with Planets, Earth, Moon, and Planets, 72, 243-249

Brandt, J.C., A'Hearn, M.F., Randall, C.E., Schleicher, D.G., Shoemaker, E.M., and Stewart, A.I.F. (1997) Small Comets (SCs): An Unstudied Population in the Solar System Inventory, in Completing the Inventory of the Solar System, ASP Conference Series, 107, T.W. Rettig and J.M. Hahn, eds., pp. 289-297

Frank, L.A., and Sigwarth, J.B. (1997a) Transient Decreases of Earth's Far-Ultraviolet Dayglow, Geophys. Res. Letters, 24, 2423-2426

Frank, L.A., and Sigwarth, J.B. (1997b) Simultaneous Observations of Transient Decreases of Earth's Far-Ultraviolet Dayglow with Two Cameras, Geophys. Res. Letters, 24, 2427-2430

Frank, L.A., and Sigwarth, J.B. (1997c) Detection of Atomic Oxygen Trails of Small Comets in the Vicinity of the Earth, Geophys. Res. Letters, 24, 2431-2434

Frank, L.A., and Sigwarth, J.B. (1997d) Trails of OH Emissions from Small Comets Near Earth, Geophys. Res. Letters, 24, 2435-2438

Hanner, M.S., Newburn, R.L., Spinrad, H., and Veeder, G.J. (1987) Comet Sugano-Saigusa-Fujikawa (1983 V) - A Small, Puzzling Comet, Astron. J., 94, 1081-1087

Lamy, P.I., Toth, I., Jorda, L., Weaver, H.A., and A'Hearn, M. (1997a) The Nucleus and Inner Coma of Comet 46P/Wirtanen, submitted to Astron. Astrophys. special Comet Wirtanen issue

Lamy, P.I., Toth, I., A'Hearn, M. and Weaver, H.A. (1997b) Hubble Space Telescope Observations of the Nucleus of Comet 45P/Honda-Mrkos-Pajdušáková and Its Inner Coma, submitted to Icarus

Marsden, B.G. (1989) The Sungrazing Comet Group. II Astron. J., 98, 2306-2321

Roemer, E. (1966) The Dimensions of Cometary Nuclei, in Nature et Origine des Comètes, Mém. Soc. Roy. Sci. Liège, Tome XII, 23-28

Yeomans, D.K. (1991) Comets, Wiley \& Sons, New York-Chichester-Brisbane-Toronto-Singapore. 